\title{
Late-Presenting Left-Sided Morgagni Congenital Diaphragmatic Hernia in a 9-Year-Old Male
}

\author{
Jennifer M. Kim, ${ }^{1}$ Marisa Couluris, ${ }^{2}$ and Bruce M. Schnapf ${ }^{2}$ \\ ${ }^{1}$ Department of Pediatrics, University of South Florida, Tampa, FL 33606, USA \\ ${ }^{2}$ Division of Pulmonary, Department of Pediatrics, University of South Florida, Tampa, FL 33606, USA
}

Correspondence should be addressed to Jennifer M. Kim, jkim@health.usf.edu

Received 30 May 2011; Accepted 20 June 2011

Academic Editors: A. Altraja, A. Celi, and N. P. Juffermans

Copyright (C) 2011 Jennifer M. Kim et al. This is an open access article distributed under the Creative Commons Attribution License, which permits unrestricted use, distribution, and reproduction in any medium, provided the original work is properly cited.

\begin{abstract}
Congenital diaphragmatic hernias are common, primarily occurring through the foramen of Bochdalek. However, in contrast, defects through the foramen of Morgagni are much more rare. When late presentations occur, patients may be asymptomatic or may be critically ill with respiratory and gastrointestinal symptoms. In this paper, we present a 9-year-old male who presented with recurrent, vague abdominal pain, and a previously normal abdominal CT scan. Initial investigation via an abdominal radiograph demonstrated an unexpected left lower lobe abnormality. Further evaluation and management revealed this abnormality to be an unusual left-sided congenital diaphragmatic hernia that appeared through the retrosternal foramen of Morgagni, a rare occurrence.
\end{abstract}

\section{Introduction}

Through the advancement of fetal ultrasound, the diagnosis of congenital diaphragmatic hernias (CDHs) typically occurs prior to delivery. However, $5-25 \%$ of cases are late presenting after the neonatal period [1-3]. CDH are relatively common, occurring in approximately 1 in 2500 births, with a majority forming posterolaterally through the foramen of Bochdalek [2]. Diaphragmatic defects retrosternally through the foramen of Morgagni are less common, occurring in only $1-5 \%$ of the patients with $\mathrm{CDH}$ [2]. These defects tend to be rightsided, in comparison to Bochdalek-type hernias, which are primarily left-sided.

We report a case of a 9 year-old male determined to have an unusual left-sided $\mathrm{CDH}$ through the retrosternal foramen of Morgagni who presented to our pediatric pulmonary service with a left lower lobe abnormality noted on imaging as part of an evaluation in the Emergency Department for abdominal pain.

\section{Case Report}

A young 9-year-old male initially presented to the Emergency Department complaining of abdominal pain for approximately 3 years with intermittent heartburn symptoms that had been worsening and were unassociated with diet, meals, or exercise. Upon obtaining an abdominal X-ray, a portion of the left lower lobe was visualized revealing an opacity (Figure 1). A chest X-ray was therefore ordered and demonstrated an irregular cystic-appearing thin-walled lucency at the anterior lung base in the left cardiophrenic sulcus and gastroesophageal junction regions (Figure 2). Given the concern for cystic anatomical lung abnormality versus possible herniation of abdominal contents on chest $\mathrm{X}$-ray, a CT scan of the chest was also obtained (Figure 3). Results demonstrated a left, anterior diaphragmatic hernia containing loops of splenic flexure through the left hemithorax. Bowel loops appeared nonobstructed, containing stool, and lung parenchyma appeared clear without inflammatory or infiltrative processes.

Review of the patient's past medical history revealed a male born full term via spontaneous vaginal delivery without complications during birth. His mother received prenatal care throughout her pregnancy. The patient was noted to have an enlarged kidney for which he had been followed as an outpatient and resolved. He developed bronchiolitis at one year of age requiring outpatient albuterol nebulizations. In addition to his most recent Emergency Department visit, he had been previously evaluated twice for abdominal pain. There was no prior history of abdominal trauma, and 


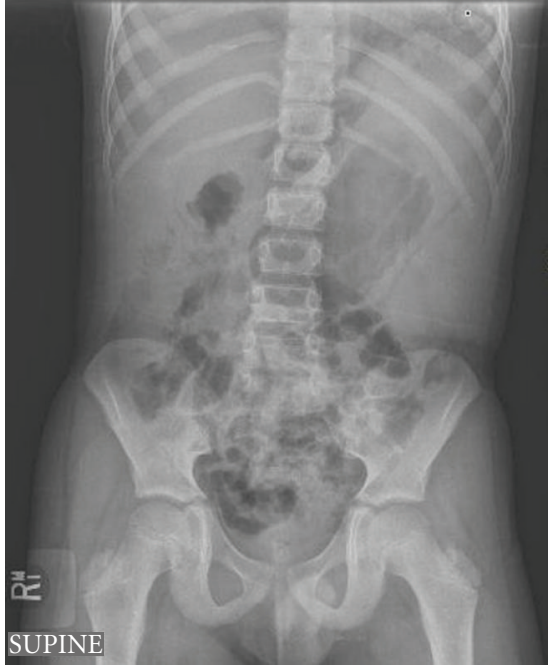

FIGURE 1

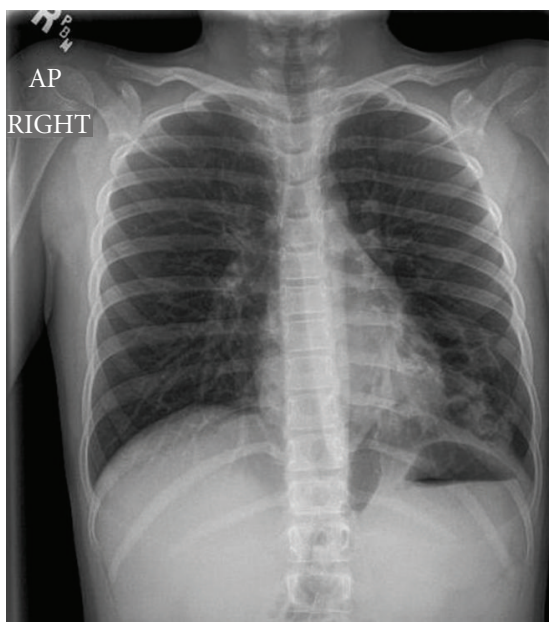

Figure 2

a previous abdominal CT scan without contrast in April 2004 was noted to be unremarkable without evidence of abdominal herniation or lung pathology.

Following evaluation by Pulmonary and Pediatric Surgery, it was determined that this needed to be surgically repaired given the risk for hemorrhage and necrosis of the bowel contents through the anterior diaphragmatic defect. The repair included celiotomy and excision of the diaphragmatic sac. A $10 \mathrm{~cm}$ by $8 \mathrm{~cm}$ defect in the anterior diaphragm with a large associated sac that was in contact with the pericardium was found in the left chest space. Gross pathological specimen of the sac was noted to be unremarkable with the finding of thin, transparent, pink fibromembranous tissue. The patient was noted to have a small apical pneumothorax postoperatively on chest Xray, requiring nasal cannula on post-op days 1 and 2 . The patient was rapidly weaned to room air without further complications and is currently asymptomatic and doing well at 6 months post-op.

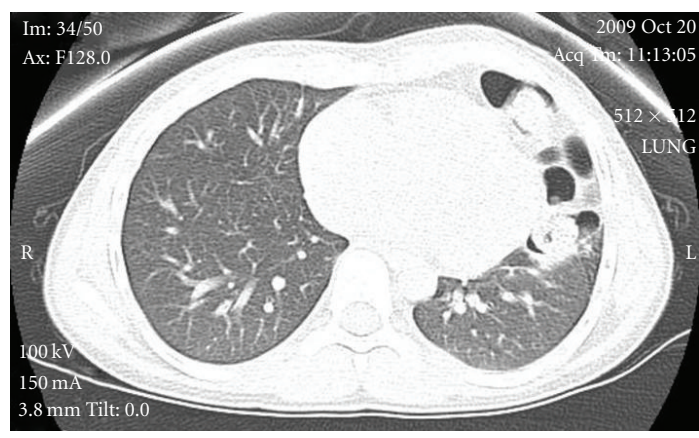

FIGURe 3

\section{Discussion}

A diaphragmatic hernia occurs when there is an anatomical defect in the diaphragm through which abdominal contents protrude into the thoracic cavity often leading to lung hypoplasia [1-4]. Approximately 1 in 2500 neonates will experience a $\mathrm{CDH}[1,2,5]$, with many being discovered prenatally or during the immediate postnatal period. These patients present with severe respiratory distress requiring emergent intubation, significant cardiorespiratory support, and surgical correction [4] in the immediate time period following birth. Protrusion of abdominal contents into the thoracic cavity forming a neonatal $\mathrm{CDH}$ is a sporadic event, but can also be late presenting occurring in infants greater than 1 month of age. In these cases, the diaphragmatic defect may not initially include herniation of abdominal contents or lung hypoplasia leading to a variable age at presentation. One study determined that the median age for this to occur was at 4 years [6] with a 2:1 male to female predominance and a left-sided occurrence. Symptoms may include emesis, nausea, abdominal pain, chest pain, dyspnea, wheezing, cough, and absent breath sounds $[5,6]$ with respiratory symptoms occurring more commonly in younger patients and gastrointestinal symptoms occurring more commonly in older patients [7]. As may have occurred with our patient with a remote history of bronchiolitis, $\mathrm{CDH}$ may easily masquerade as a respiratory disorder.

Chest X-ray is the most common imaging utilized to diagnose this type of $\mathrm{CDH}$, although many patients may have initial negative diagnostic imaging [8]. A bowel gas pattern, air-fluid level in the thoracic cavity, or lucency in the chest with deviation of the heart and mediastinum may be observed, making the final diagnosis complicated and challenging $[1,4,6]$. Many patients are misdiagnosed initially secondary to the constellation of vague symptoms and X-ray findings; however, sensitivity may be increased to approximately $75 \%$ via insertion of a nasogastric tube $[5,9]$. Common misdiagnoses include pneumothorax, pneumonia, pleural effusion, infectious etiology, or cystic malformation [9-13]. If CDH is suspected upon evaluation, an ultrasound of the chest may also be useful [9] with further diagnostic imaging including CT scan, MRI, or upper and lower GI contrast studies. 
The most common type of $\mathrm{CDH}$ reported is through the posterolateral foramen of Bochdalek with $80 \%$ occurring on the left side $[1,6]$. In comparison, only $1-5 \%$ of $\mathrm{CDH}$ occur through the anterior foramen of Morgagni [2], which is retrosternal at the sternocostal trigone, known as the space of Larrey [14]. Bochdalek-type CDHs typically occur on the left, whereas Morgagni-type $\mathrm{CDHs}$ are primarily right-sided $[1,2]$. Although the underlying etiology of $\mathrm{CDH}$ is unknown, the type of diaphragmatic herniation created is likely secondary to the embryological formation occurring in the eight to tenth weeks of fetal development. During this time, the coelomic cavity divides into the thoracic and abdominal cavities, and the elongated, rotated gastrointestinal tract migrates back into the abdomen.

Defects in the diaphragm through the foramen of Morgagni, as seen in our patient, are rare and occur when there is a lack of fusion between both the fibrotendinous portion of the pars sternalis and the costochondral arches [2]. Ninety percent of these defects are right-sided [2, 15], in contrast to our patient, whose defect occurred primarily on the left side. Left-sided defects are thought to be less common secondary to the pericardial attachment to the diaphragm, which provides more support [15]. A small, three-center study in Turkey between 1994 and 1999 found 7 patients with Morgagni CDH diagnosed by chest X-ray and confirmed by barium-contrast radiographs. Six of these patients had a right-sided defect, whereas only 1 patient had a left-sided defect [16]. Additionally, a large, multicenter study by the Congenital Diaphragmatic Hernia Study Group in 2005 found $69 \%$ (53 out of 79) of hernia cases through the foramen of Bochdalek had left-sided defects, whereas $43 \%$ of hernias ( 3 out of 7 ) occurring through the foramen of Morgagni were right-sided [7]. None of the Morgagni hernias identified in the study group occurred on the left side. In a 2007 study published by Al-Salem in Saudi Arabia, review of records over an 18-year period revealed only 20 cases of $\mathrm{CDH}$ through the foramen of Morgagni. Of these 20 patients, only 5 suffered from a left-sided defect (25\%), whereas 9 had a right-sided defect $(45 \%)$ and 6 were found to be bilateral (30\%) [15]. These studies further demonstrate the rare occurrence of congenital Morgagni hernias, especially those occurring on the left side.

Although there is no known genetic cause of Morgagni hernias, they are often seen in conjunction with other congenital defects such as heart defects and Down's syndrome [16]. The transverse colon and omentum commonly herniate into the sac with two studies identifying a sac in $95 \%$ of patients $[2,15]$. Despite a significant defect and herniation of abdominal contents most patients remain asymptomatic until the diagnosis is made via radiological imaging. Management of patients with late-presenting $\mathrm{CDH}$ of either the Bochdalek or Morgagni type consists of stabilization and prompt surgical repair. When the correct diagnosis is made, survival is excellent, between 97 and 100\%, in comparison to neonatal $\mathrm{CDH}$ [4]. Patients typically have uncomplicated recovery courses and very few sequelae. Although delay in treatment and surgical correction is controversial [2], it is generally felt that delayed intervention leads to necrosis of the bowel and bowel obstruction [6].

\section{Conclusion}

We have described an interesting case of a young male initially presenting with abdominal pain and radiological imaging noting a rare, left, anterior $\mathrm{CDH}$ through the foramen of Morgagni. Surgical correction of the anterior defect and pathological evaluation confirmed the diagnosis. This case illustrates the relatively asymptomatic or vague symptoms at presentation that may be associated with late-presenting $\mathrm{CDH}$ with previously normal radiological imaging studies. In such patients, consideration of more rare etiologies, such as herniation of abdominal contents into the intrathoracic space, should be strongly considered.

\section{References}

[1] F. C. Vandy, J. E. Landrum, N. R. Gerig, and J. A. Prahlow, "Death due to late-presenting congenital diaphragmatic hernia in a 2-year-old child," American Journal of Forensic Medicine and Pathology, vol. 29, no. 1, pp. 75-79, 2008.

[2] S. Singh, M. S. Bhende, and J. M. Kinnane, "Delayed presentations of congenital diaphragmatic hernia," Pediatric Emergency Care, vol. 17, no. 4, pp. 269-271, 2001.

[3] V. S. Jones and R. C. Cohen, "What causes intrathoracic gastric dilatation in a late-presenting diaphragmatic hernia?" Pediatric Emergency Care, vol. 24, no. 5, pp. 317-318, 2008.

[4] M. M. Blackstone and R. D. Mistry, "Late-presenting congenital diaphragmatic hernia mimicking bronchiolitis," Pediatric Emergency Care, vol. 23, no. 9, pp. 653-656, 2007.

[5] J. Nirgiotis, "Late presentation of congenital diaphragmatic hernia," Archives of Pediatrics \& Adolescent Medicine, vol. 163, no. 6, p. 584, 2009.

[6] M. Waseem and F. Quee, "A wheezing child breath sounds or bowel sounds?" Pediatric Emergency Care, vol. 24, no. 5, pp. 304-306, 2008.

[7] Congenital Diaphragmatic Hernia Study Group, "Latepresenting congenital diaphragmatic hernia," Journal of Pediatric Surgery, vol. 40, no. 12, pp. 1839-1843, 2005.

[8] L. Berman, D. Stringer, S. H. Ein, and B. Shandling, "The late-presenting pediatric Bochdalek hernia: a 20-year review," Journal of Pediatric Surgery, vol. 23, no. 8, pp. 735-739, 1988.

[9] M. E. Coren, M. Rosenthal, and A. Bush, "Congenital diaphragmatic hernia misdiagnosed as tension pneumothorax," Pediatric Pulmonology, vol. 24, no. 2, pp. 119-121, 1997.

[10] E. Picard, A. B. Nun, D. Fisher, S. Schwartz, M. Goldberg, and S. Goldberg, "Morgagni hernia mimicking pneumonia in Down syndrome," Journal of Pediatric Surgery, vol. 42, no. 9, pp. 1608-1611, 2007.

[11] S. Harte, R. G. Casey, D. Mannion, and M. Corbally, "When is a pneumothorax not a pneumothorax?" Journal of Pediatric Surgery, vol. 40, no. 3, pp. 586-587, 2005.

[12] Y. Kadian, K. Rattan, M. Verma, and P. Kajal, "Congenital diaphragmatic hernia: misdiagnosis in adolescence," Journal of Indian Association of Pediatric Surgeons, vol. 14, no. 1, pp. 31-33, 2009.

[13] U. Zaleska-Dorobisz, M. Baglaj, D. Sokolowska, and J. Ladogorska, "Late presenting diaphragmatic hernia: clinical and diagnostic aspects," Medical Science Monitor, vol. 13, no. 10, pp. 137-146, 2007.

[14] B. L. Robinson and D. M. Shahian, "Transthoracic repair of an unsuspected left foramen of morgagni hernia," Annals of Thoracic Surgery, vol. 86, no. 5, pp. 1693-1695, 2008. 
[15] A. H. Al-Salem, "Congenital hernia of Morgagni in infants and children," Journal of Pediatric Surgery, vol. 42, no. 9, pp. 15391543, 2007.

[16] H. Soylu, U. Koltuksuz, N. O. Kutlu et al., "Morgani hernia: an unexpected cause of respiratory complaints and a chest mass," Pediatric Pulmonology, vol. 30, no. 5, pp. 429-433, 2000. 


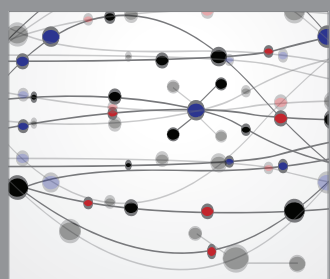

The Scientific World Journal
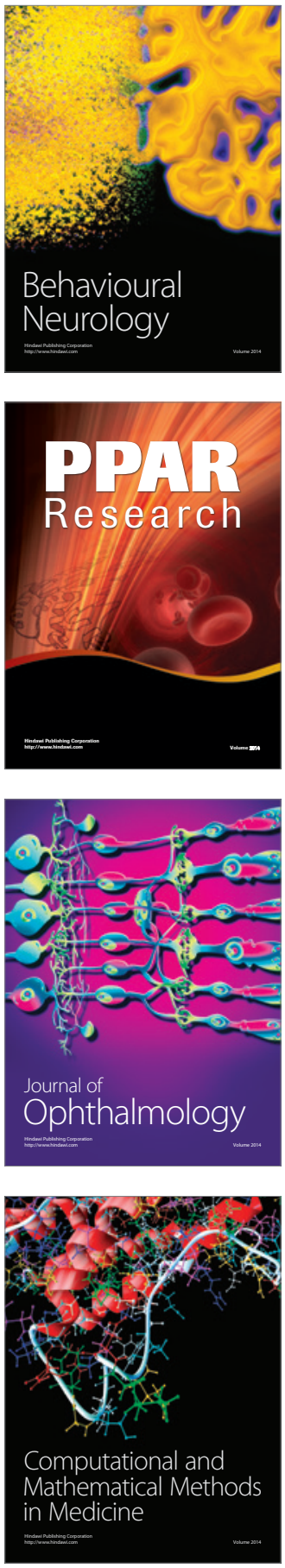

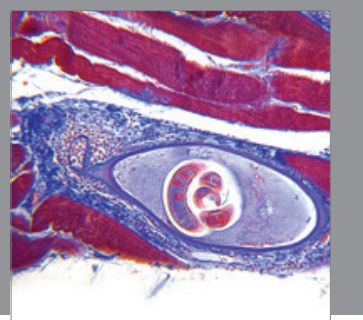

Gastroenterology

Research and Practice
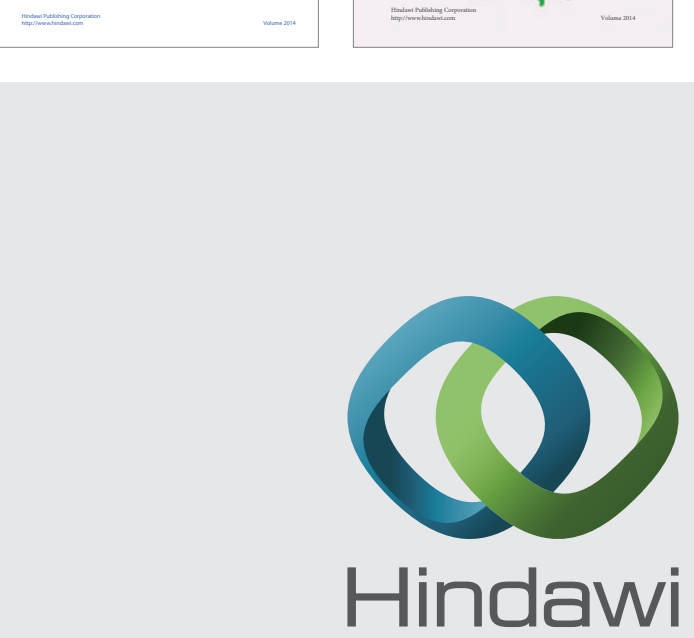

Submit your manuscripts at

http://www.hindawi.com
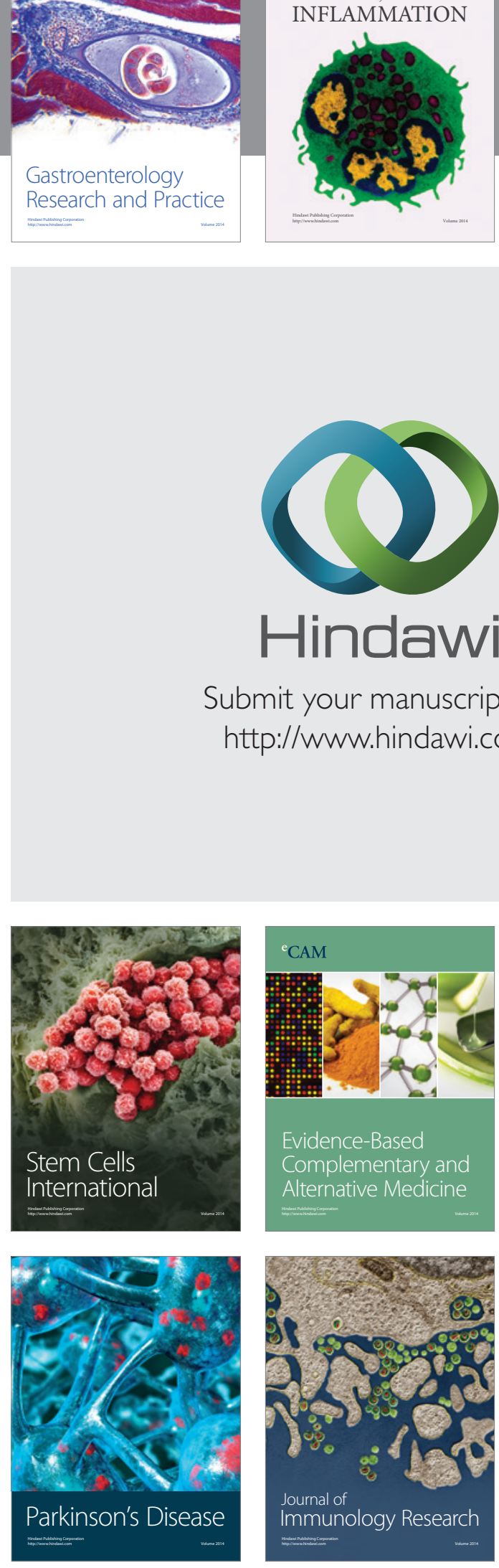

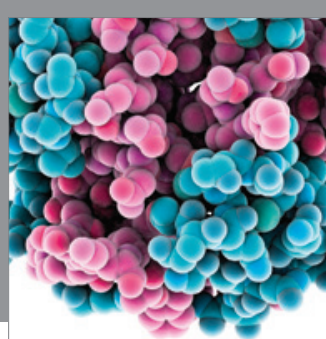

Diabetes Research
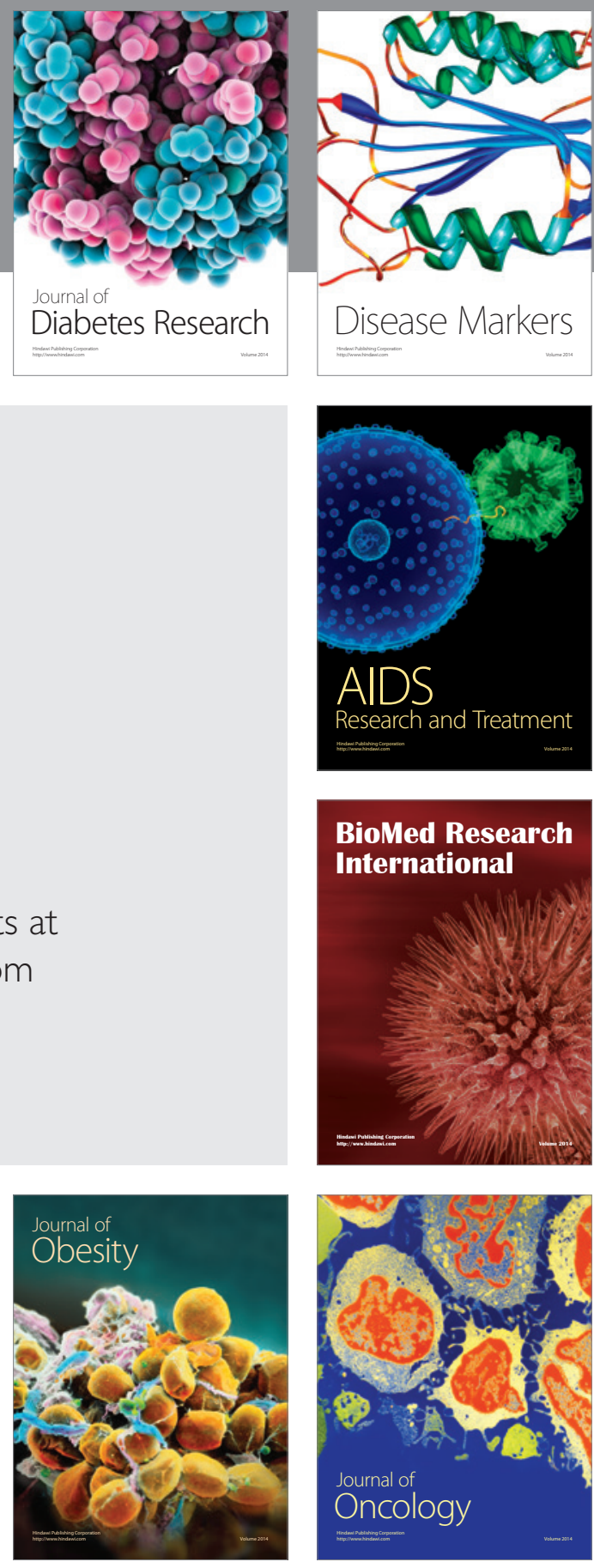

Disease Markers

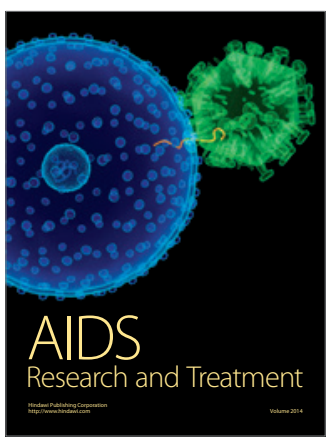

BioMed Research

International
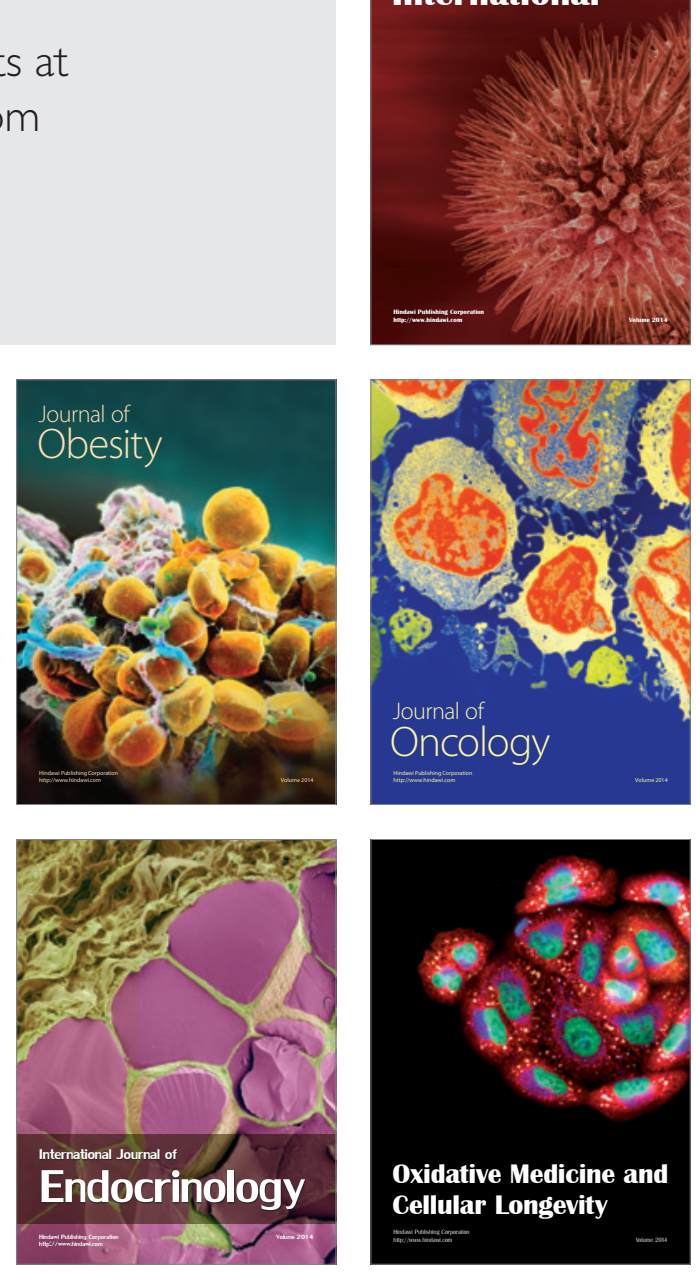